\title{
Evolutionary Particularities in a Case of Severe Pneumonia in Children - Case Report
}

Marcela Daniela IONESCU ${ }^{a}$,, Nicoleta Aurelia POPESCU ${ }^{a}$, Georgiana BALANª, Veronica MARCUc, Augustina ENCULESCU ${ }^{e}$, Lorena VATRA ${ }^{f}$, Marcel OANCEA ${ }^{f}$, Mihaela BALGRADEAN ${ }^{b}, \mathrm{~d}$

${ }^{a}{ }^{\text {st }}$ Pediatric Department, "MS Curie" Emergency Children's Hospital, Bucharest, Romania

${ }^{\text {b }}{ }^{\text {nd }}$ Pediatric Department, "MS Curie" Emergency Children's Hospital, Bucharest, Romania

'Radiology Department, "MS Curie" Emergency Children's Hospital, Bucharest, Romania d"Carol Davila" University of Medicine and Pharmacy, Bucharest, Romania ePathology Department,"MS Curie" Emergency Children's Hospital, Bucharest, Romania fPediatric Surgery Department, "MS Curie" Emergency Children's Hospital, Bucharest, Romania

\begin{abstract}
-ABSTRACT
Necrotizing pneumonia remains an uncommon complication of pneumonia in children, but its incidence is increasing. Pneumococcal infection is the predominant cause of severe necrotizing pneumonia in children, but methicillin resistant Staphylococcus aureus (MRSA) and Panton-Valentine leukocidin (PVL) staphylococcal infections are also important.

We present the case of a four-year-old girl,with an unremarkable medical history, who was admitted in our hospital with a history of high fever, productive cough and tachypnea lasting for 10 days, progressive worsening despite empirical oral antibiotic. Following physical examination, laboratory investigations and thoracic radiography, we established the diagnosis of left lower lobe pneumonia with parapneumonic effusion, acute respiratory failure and sepsis. Medical treatment with systemic antibiotics was initiated, but the evolution was unfavorable. Seriated chest $X$-rays and also high resolution computed tomography with contrast of the lung were performed, revealing the progression to extensive necrotizing pneumonia with multiple cystic lesions causing right mediastinal deflection. The parenteral broad spectrum antibiotic regimen was adjusted, still with unfavorable evolution, requiring surgical treatment (left inferior lobectomy and pleural draining). Postoperatively, recovery was uneventful. The patient was discharged with clinical and laboratory improvement of his condition, a repeated chest X-ray showing good expansion of upper left parenchyma.
\end{abstract}

Keywords: necrotizing pneumonia, parapneumonic pleural effusion, high resolution computed tomography, pneumococcal infection.

\footnotetext{
Address for correspondence:

Dr. Marcela Daniela Ionescu "MS Curie" Emergency Children's Hospital Tel.: 0741220 099;

Email: drmarcela.ionescu@gmail.com
} 


\section{INTRODUCTION}

N ecrotizing pneumonia (NP) is a rare and severe complication of bacterial community-acquired pneumonia (CAP) and is characterized by liquefaction and cavitation of lung tissue (1). Lying on a spectrum of lung abscess and pulmonary gangrene, NP is characterized by pulmonary inflammation with consolidation, peripheral necrosis and multiple small cavities (2). The necrotic process can occur in any lobe of the lung, but the involvement of lower lobes is more frequent. The pathogenetic mechanisms of NP are not clear, but it is commonly believed that tissue necrosis occurs as a result of an inflammatory response due to toxins produced by the invasive pathogen or the associated vasculitis with thrombotic occlusion of alveolar capillaries $(1,3,4)$.

The onset of the disease is usually marked by the development of typical signs and symptoms of pneumonia. Children usually present high fever, cough, and tachypnea lasting for several days.

The physical examination is similar to uncomplicated pneumonia with fever, tachypnea, decreased breath sound, isolated crackles (5). Most patients with necrotic disease have already been treated with antibiotics. NP should be suspected when a patient with pneumonia develops progressive respiratory distress, hemoptysis or septic shock, despite appropriate antibiotic treatment. Physical examination may often reveal signs of pleural effusion (1, 4-6).

Chest radiography can detect the diagnosis of $\mathrm{NP}$, but sometimes, the presence of pleural effusion may obscure the underlying lung process. Radiographic criteria for necrotizing pneumonia include loss of the normal lung architecture and presence of areas of decreased parenchymal enhancement, representing liquefaction, which is progressively replaced by multiple small air or fluid-filled cavities. Un upright chest radiograph may show an air-fluid level in the pleural space $(1,4,7)$. The process may further extend to the pleural space and create a bronchopleural fistula, especially when the necrotic segment is adjacent to the pleural surface $(2,8)$.

Chest computed tomography with contrast is helpful for a more definitive diagnosis when NP is suspected, and it may highlight multiple cavitary lesions within the consolidation $(1,4,5)$.
Blood acute reactants such as white cell count, C-reactive protein, erythrocyte sedimentation rate and procalcitonin are increased. The pleural fluid characteristics associated with NP reflect those usually found in parapneumonic effusion or empyema. Blood cultures should be obtained despite the low isolation rate (10-22\%).

$S$. pneumoniae is the more frequent etiological agent of NP. Mycoplasma pneumoniae and S. aureus strains, often methicillin-resistant, producing cytotoxin Panton-Valentine leukocidine, have also been involved in the genesis of necrotizing pneumonia. Other bacteria, less frequently reported, include $S$. pyogenes, $S$. viridans, $P$. aeruginosa and anaerobes (9-13).

Almost $50 \%$ of the cases of NP have no identified etiological cause with common microbiological methods. New methods, such as PCR analysis, could potentially increase the diagnostic $(1,4)$.

There is not a general agreement on the best therapeutic strategy for NP. The focus of initial management is on early initiation of appropriate empirical antibiotics and a careful attempt to identify the pathogen $(1,4,8)$.

An exclusive treatment with high-dose antibiotics is frequently successful in children, with unexpected parenchymal conservation and lung re-expansion (1, 2, 4). As for empyema, the choice of initial antibiotics should be directed at broad coverage of commonly implicated pathogens. Supportive therapy is a key element because many children are critically ill.

Serial chest $\mathrm{x}$-ray or thoracic ultrasonography examinations are useful for identifying the development of pleural effusions $(2,3,7)$. Parapneumonic pleural fluid should be sampled and drained if pus or bacteria are present if it is loculated or the $\mathrm{pH}$ is lower than 7.2.

A chest CT scan with contrast is also useful to look for pleural thickening and enhancement suggestive of empyema and to evaluate multiseptated or loculated effusion that may require surgical intervention $(1,4,7)$.

Intrapleural fibrinolysis may potentially involve a risk of NP. Surgery VATS has been successfully used in children with NP and should be reserved for patients with associated empyema or to resolve bronchopleural fistulae that do not close with conservative treatment. Surgical resection of the lung should be reserved for particularly severe cases $(1,2,4,8)$. 
Long-term outcome for children with NP is usually good.

\section{CASE REPORT}

A pre previously healthy four-year-old girl was admitted in our clinic with a history of high fever, productive cough and tachypnea lasting for 10 days, progressive worsening despite empirical oral antibiotic (Clarithromycin) and symptomatic treatment given by the general physician.

Physical examination upon presentation revealed an underweight child, with high fever $\left(40^{\circ} \mathrm{C}\right)$ and lethargic aspect, in moderate respiratory distress, with tachypnea (RR $44 \mathrm{rpm}$ ), retractions, oxygen desaturation $(90 \%$ in room air) and persistent productive cough. Dullness to percussion and decreased respiratory sounds with crackles in the lower left lung field were detected. The child was tachycardic (HR 140 bpm), with normal blood pressure, and she complained of left upper quadrant abdominal pain. The rest of clinical examination was unremarkable.

Laboratory investigations showed leukocytosis (WBC 42.000/ $\mu \mathrm{L}$ ) with neutrophilia $(82.8 \%)$, highly elevated inflammatory tests (CRP $403 \mathrm{mg} / \mathrm{L}$, procalcitonin $>10 \mathrm{ng} / \mathrm{mL}$ ), mild normocytic normochromic anemia (Hb $10 \mathrm{~g} / \mathrm{dL}, \mathrm{MCV} 84 \mathrm{fL}$, $\mathrm{MCH} 26 \mathrm{pg}$ ), while other blood tests were normal (including negative blood cultures).

Chest radiograph revealed large opacity in the inferior half of the left hemithorax suggestive

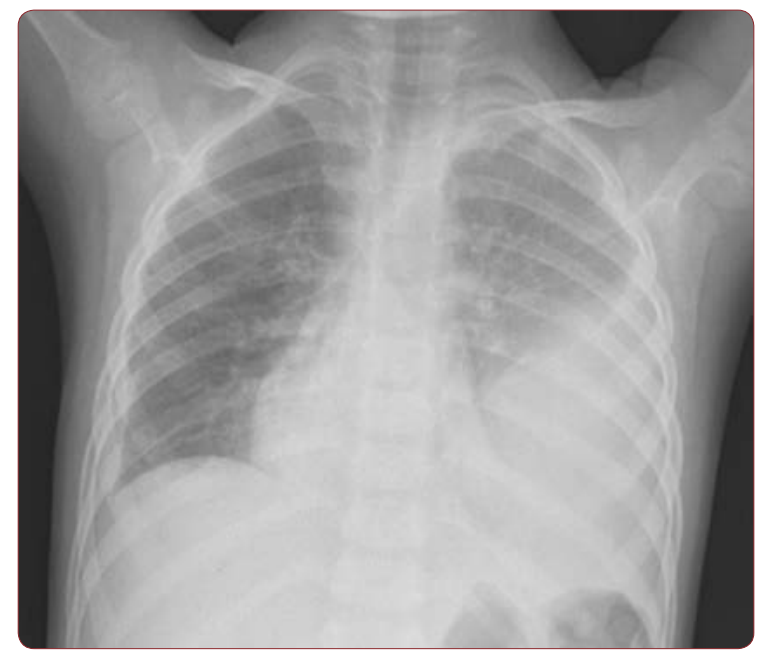

FIGURE 1. Large homogenous opacity with costal intensity and net contour occupying the inferior half of the left hemithorax, suggestive of pulmonary condensation associated with pleural effusion. Minimal right latero-basal pleural reaction. of pneumonia associated with pleural effusion and minimal right latero-basal pleural reaction (Figure 1). Thoracic ultrasonography confirmed parenchymal consolidation with pleural effusion $(10 \mathrm{~mm})$. The diagnosis was left lower lobe pneumonia with parapneumonic effusion.

Medical treatment with antibiotics (Meropenem and Vancomycin), endovenous perfusion and oxygen supplementation were initiated. The patient maintained a satisfactory general state, with mild clinical and laboratory improvement, moderate fever, reduction of respiratory distress, but hyper-resonant left inferior hemithorax with an abolished vesicular murmur, and crackles in this area occurred.
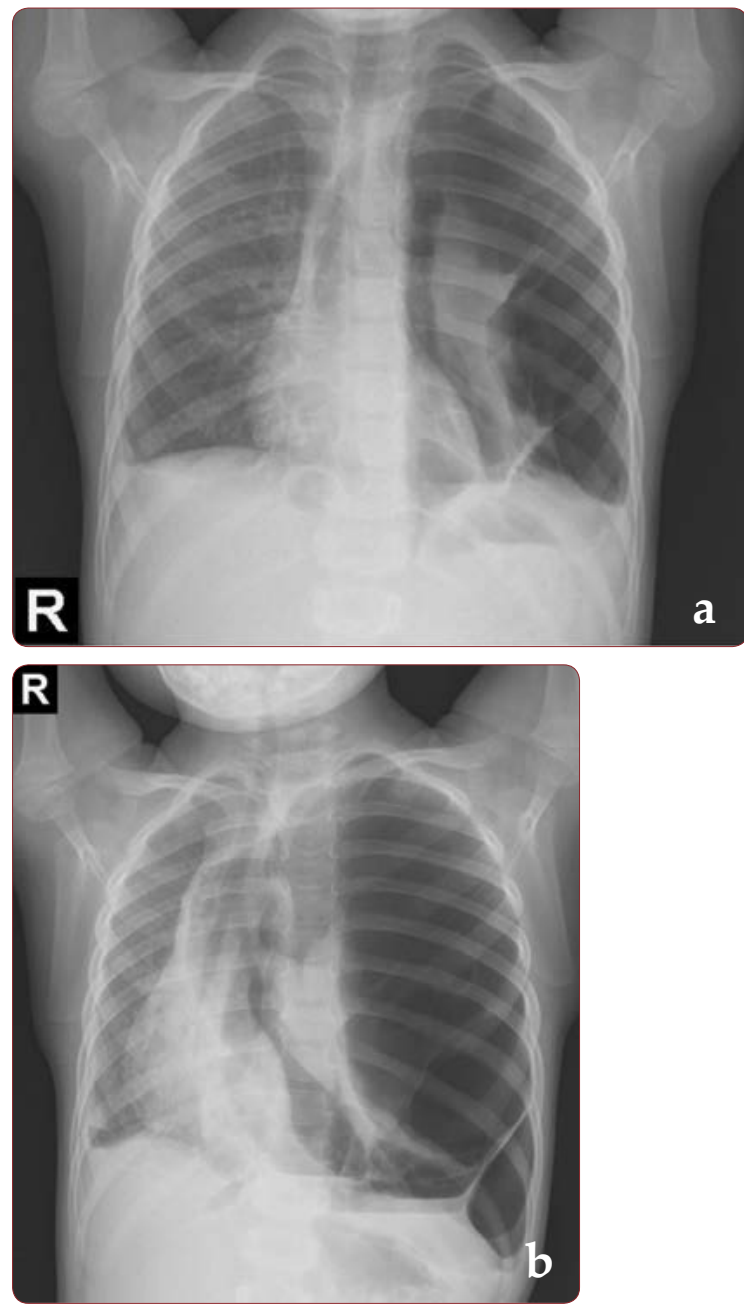

FIGURE 2. a) Pulmonary hyper-transparency with lack of interstitial drawing in the inferior half of left lung. Latero-basal opacity in the right side (suggestive for pleural effusion); b) Pogression of the hyper-transparency image, with displacement of entire mediastinum to the right side and left pulmonary parenchymal collapse. 


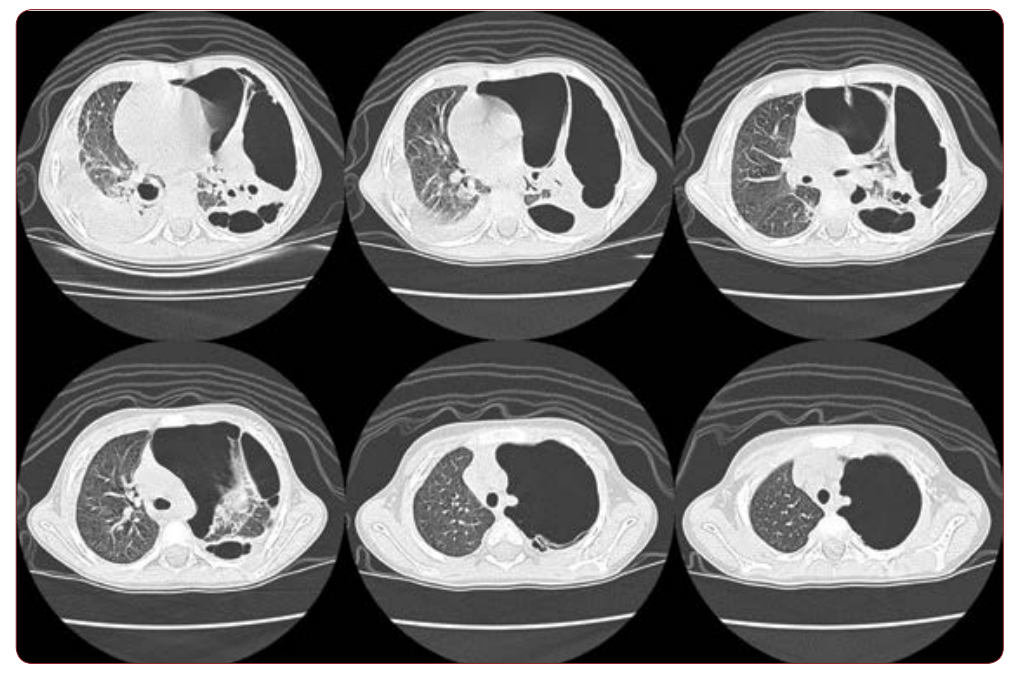

FIGURE 3. Profound alteration of the left pulmonary architecture, through the presence of numerous cystic air-filled lesions, with thin walls and collapsed parenchyma among them, inducing right cord and mediastinum and deflection.

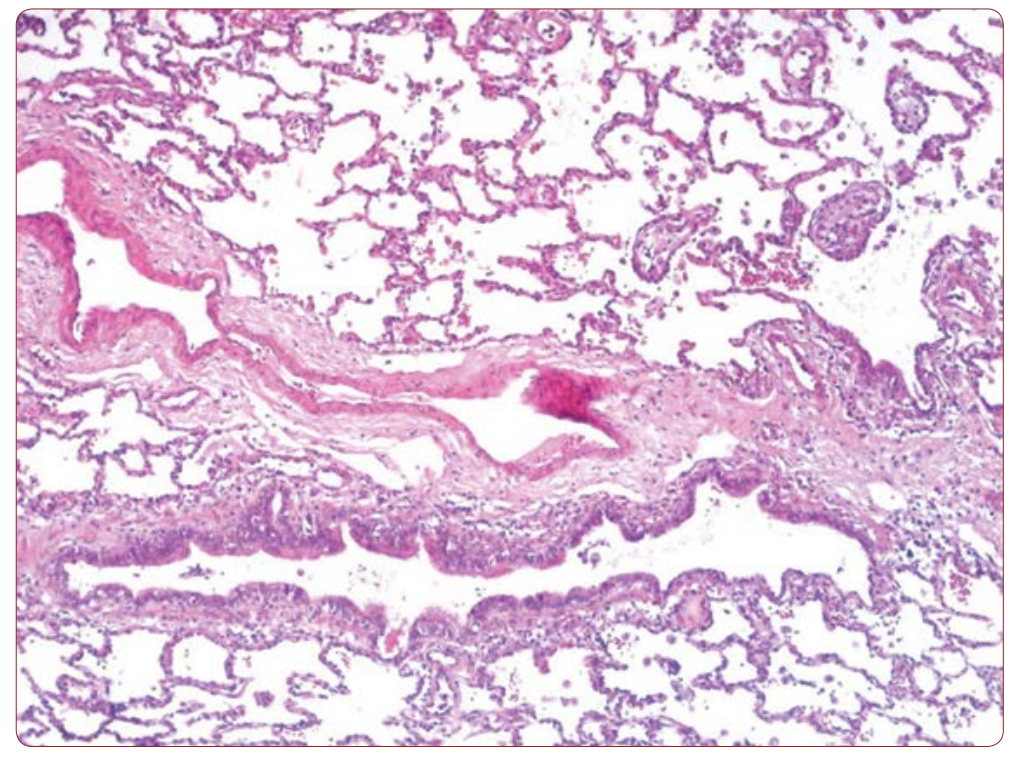

FIGURE 4. Col.HE, Ob.10X. Mixed inflammatory infiltration. Frequent intra-alveolar macrophages. Abscess areas with central necrosis and circumscribed by granulation tissue. Emphysematous areas with alveolar space distention.

Therefore, seriated chest X-rays were performed. Pulmonary hyper-transparency with lack of interstitial drawing in the inferior half of left lung was first noted on day 7 (Figure 2a). The parenteral antibiotic regimen was adjusted by adding Clindamycin and Fluconazole, still with unfavorable evolution. On physical examination, she associated thoracic asymmetry, diminished respiratory movements on the left side and recurrence of respiratory distress. Repeated chest $X$-ray on day 21 revealed the progression of the hyper-transparency image occupying the entire left lung, with displacement of the mediastinum to the right side and left pulmonary parenchymal collapse (Figure 2b).

HRCT of the lung was performed and showed profound alteration of the left pulmonary architecture through the presence of numerous cystic air-filled lesions, with thin walls and collapsed parenchyma among them, inducing right mediastinum deflection (Figure 3). The diagnosis of acute left inferior lobe bacterial pneumonia complicated with severe necrotizing pneumonia was established. The pediatric surgery department was consulted for the possibility of surgical treatment. The patient underwent left anterolateral thoracotomy, left inferior lobectomy and pleural draining.

The histopathological examination confirmed necrotizing pneumonia. It also excluded a cystic adenomatous malformation underlying the infectious process (Figure 4).

Postoperative, the recovery was uneventful, and the patient was discharged on day 31 of admission. A follow-up chest X-ray six months later shows almost complete resolution (Figure 5). 


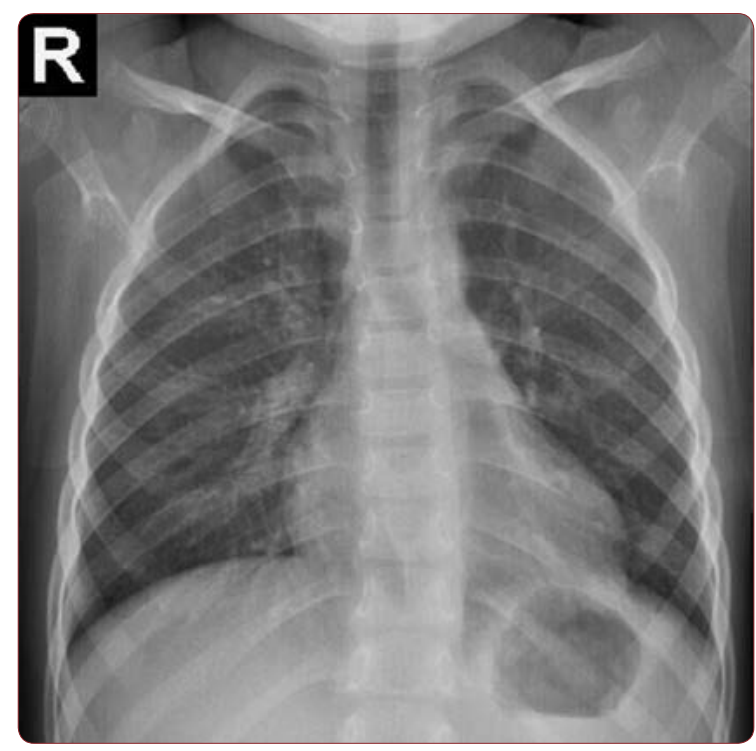

FIGURE 5. Good expansion of upper left parenchyma.

\section{DISCUSSION}

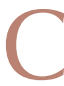
hildren with NP have similar presenting signs and symptoms as uncomplicated pneumonia. Their symptoms may rapidly progress despite appropriate antibiotic treatment. We were confronted with a case of community-acquired pneumonia in an immunocompetent child, with an unfavorable progression to extensive NP with multiple cystic lesions causing mediastinal displacement.

Possible complications of community-acquired pneumonia were discussed and the following differential diagnoses were considered: primary or secondary lung abscess (occurring in a predisposing context, possibly superinfected congenital malformation), necrotizing pneumonia or pneumatocele. Immunodeficiency syndromes, hyper-IgE syndrome and pulmonary tuberculosis were excluded (normal immunogram, normal IgE, negative tuberculin skin test).

Postoperatively intravenous antibiotics are continued and the patient's evolution was good. A follow up chest radiograph six months later shows almost complete resolution. There are no practice guidelines to direct the care of patients with NP. Establishing the need and also the right moment for surgery is still a challenge.

Conflicts of interest: none declared. Financial support: none declared.

\section{$\boldsymbol{R}_{\text {FFERENCES }}$}

1. Chatha N, Fortin D, Bosma KJ. Management of necrotizing pneumonia and pulmonary gangrene: A case series and review of the literature. Canadian Respiratory Journal 2014;4:239.

2. Master IB, Isles AF, Grimwood K. Necrotizing pneumonia: an emerging problem in children? Pneumonia (Nathan) 2017;9:11.

3. Tsay $\mathbf{Y F}, \mathbf{K u} \mathbf{Y H}$. Necrotizing pneumonia: a rare complication of pneumonia requiring special consideration. Curr Opin Pulm Med 2012;3:246-252.

4. de Benedicts FM, Azzari Chiara, Bernardi F. Pleural infection, necrotising pneumonia, and lung abscess, In: Eber E, Midulla F, Paediatric Respiratory Medicine, European Respiratory Society, 2013, pp 376-382.

5. Sawicki GS, Lu FL, Valim C, Cleveland RH, ColinAA. Necrotising pneumonia is an increasingly detected complication of pneumonia in children. European
Respiratory Journal 2008;31:1285-1291.

6. Hacimustafaoglu $M$, Celebi S, Sarimehmet H, Gurpinar A, et al. Necrotizing pneumonia in children. Acta Pediatrica 2004;9:1172-1177.

7. Light MJ, Oneto Julieta M. Pulmonary Imaging. In: Light MJ, Blaisdell CJ, Homnick DN, Pediatric Pulmonology, American Academy of Pediatrics, Miami, 2011, pp 148-151.

8. Steinfeld Jonathan. The complications of Pneumonia. In: Light MJ, Blaisdell CJ, Homnick DN, Pediatric Pulmonology, American Academy of Pediatrics, Miami, 2011, pp 423-443.

9. Labandeira-Rey Maria, Couzon Florence, Boisset Sandrine, Brown EL, et al. Staphylococcus aureus Panton-Valentine Leukocidin Causes Necrotizing Pneumonia. Science 2007;5815:1130-1133.

10. Gillet Y, Issartel B, Varhems P, Fournet JC, Lina G. Association between Staphylococ- cus aureus strains carrying gene for Panton-Valentine leukocidin and highly lethal necrotising pneumonia in young immunocompetent patients. Lancet 2002;9308:753-759.

11. Hsieh Y-C, Hsiao C-H, Tsao P-N, Hsueh P-R, et al. Necrotizing pneumococcal pneumonia in children: The role of pulmonary gangrene. Pediatric Pulmonology 2006;7:623-629.

12. Hsieh Y-C, Hsueh P-R, Lu C-Y, Lee P-I, et al. Clinical Manifestations and Molecular Epidemiology of Necrotizing Pneumonia and Empyema Caused by Streptococcus pneumoniae in Children in Taiwan. Clinical Infectious Diseases 2004;6:830-835.

13. Lemaître Chloé, Angoulvant $F$, Gabor $F$, Makhoul Juliette, et al. Necrotizing Pneumonia in Children: Report of 41 Cases Between 2006 and 2011 in a French Tertiary Care Center. The Pediatric Infectious Disease Journal 2013;10:1146-1149. 